\title{
High-Fat Diet-Induced IL-17A Exacerbates Psoriasiform Dermatitis in a Mouse Model of Steatohepatitis
}

Philippe Vasseur, ${ }^{* \dagger}$ Laura Serres, ${ }^{* \dagger}{ }^{\dagger}$ Jean-François Jégou, ${ }^{\dagger}$ Mathilde Pohin, ${ }^{\dagger}$ Adriana Delwail ${ }^{\ddagger}{ }^{\ddagger}$ Isabelle Petit-Paris, ${ }^{\dagger}$ Pierre Levillain, ${ }^{\oplus}$ Laure Favot, ${ }^{\dagger}$ Michel Samson, ${ }^{*}$ Hans Yssel, ${ }^{* *}$ Franck Morel, $^{\dagger}$ Christine Silvain, ${ }^{* \dagger}$ and Jean-Claude Lecron ${ }^{\dagger, \dagger \dagger}$

From the Departments of Hepatogastroenterology, ${ }^{*}$ Pathology, "and Immunology/Inflammation, ${ }^{\dagger \dagger}$ Poitiers University Hospital, Poitiers; the Laboratory of Inflammation and Epithelial and Cytokine Tissues EA 4331, ${ }^{\dagger}$ ImageUP, ${ }^{\ddagger}$ and the STIM Laboratory, ${ }^{\S}$ CNRS ERL 7368, Poitiers University, Poitiers; the IRSET, Inserm U1085, University of Rennes 1, Rennes; and the Sorbonne University, ** UPMC Univ Paris 06, CIMI, Inserm U1135, Paris, France

Accepted for publication

May 17, 2016.

Address correspondence to Philippe Vasseur, M.D., Ph.D., Laboratoire Inflammation, Tissus Epithéliaux et Cytokines (EA 4331), Pôle Biologie Santé B36/B37, 1 rue Georges Bonnet, TSA 51106, 86073 Poitiers Cedex 9, France. E-mail: vasseur.philippe@chnds.fr.

\begin{abstract}
Recent studies suggest that psoriasis may be more severe in patients with nonalcoholic fatty liver disease, particularly in those with the inflammatory stage of steatohepatitis [nonalcoholic steatohepatitis (NASH)]. Herein, we investigated the impact of diet-induced steatohepatitis on the severity of imiquimod-induced psoriasiform dermatitis. Mice fed with a high-fat diet developed steatohepatitis reminiscent of human NASH with ballooning hepatocytes and significant liver fibrosis. Mice with steatohepatitis also displayed moderate cutaneous inflammation characterized by erythema, dermal infiltrates of CD45 leukocytes, and a local production of IL-17A. Moreover, steatohepatitis was associated with an epidermal activation of caspase- 1 and cutaneous overexpression of IL-1 $\beta$. Imiquimod-induced psoriasiform dermatitis was exacerbated in mice with steatohepatitis as compared to animals fed with a standard diet. Scale formation and acanthosis were aggravated, in correlation with increased IL-17A and IL-22 expression in inflamed skins. Finally, intradermal injection of IL-17A in standard diet-fed mice recapitulated the cutaneous pathology of mice with steatohepatitis. The results show that high-fat diet-induced steatohepatitis aggravates the inflammation in psoriasiform dermatitis, via the cutaneous production of IL-17A. In agreement with clinical data, this description of a novel extrahepatic manifestation of NASH should sensitize dermatologists to the screening and the management of fatty liver in psoriatic patients. (Am J Pathol 2016, 186: 2292-2301; http://dx.doi.org/10.1016/j.ajpath.2016.05.012)
\end{abstract}

Nonalcoholic fatty liver disease (NAFLD) has emerged as a leading cause of cirrhosis and hepatocellular carcinoma in Western countries. Its spectrum encompasses the initial stage of liver steatosis, evolving into nonalcoholic steatohepatitis (NASH), liver fibrosis, and cirrhosis. Increasing attention is being given to the extrahepatic complications observed in patients with NAFLD, such as cardiovascular diseases, type 2 diabetes mellitus, or colorectal cancer. ${ }^{1}$ Recently, a positive association between NAFLD and psoriasis has been reported. The susceptibility of psoriatic patients to develop NAFLD is increased, ${ }^{2}$ whereas the presence of NAFLD itself may aggravate the severity of psoriasis. ${ }^{3,4}$ These observations emphasize the reciprocal deleterious action of the psoriatic inflammatory process and NAFLD in which cytokines play a pivotal role. During NAFLD, progression from steatosis to steatohepatitis is facilitated by tumor necrosis factor- $\alpha$ and IL-6, whereas transforming growth factor- $\beta$ favors evolution toward liver fibrosis, resulting from the activation of hepatic stellate cells. ${ }^{5}$ An early event in the pathogenesis of psoriasis involves the polarization of naïve $\mathrm{T}$ lymphocytes into $\mathrm{T}$ helper (Th) 17 and Th1 lymphocytes that secrete IL-17, IL22 , tumor necrosis factor- $\alpha$, and interferon- $\gamma$, respectively, ${ }^{6}$

Supported by University of Poitiers, Poitiers University Hospital, Nord Deux-Sèvres Hospital, and Région Poitou-Charentes.

C.S. and J.-C.L. contributed equally as senior authors.

The research was performed in the Laboratory of Inflammation and Epithelial and Cytokine Tissues (Poitiers University).

Disclosures: None declared.

Current address of P.V., Department of Hepatogastroenterology, Nord Deux-Sèvres Hospital, Thouars, France. 
thereby conferring a characteristic cytokine profile to skin lesions. ${ }^{7,8}$ As a result of excessive local inflammation, psoriasis and NASH are associated with a systemic inflammatory process, characterized by increased C-reactive protein serum levels. ${ }^{9,10}$ This proinflammatory state may have important clinical implications. For example, the risk of myocardial infarction is increased in both disorders independently of cardiovascular risk factors. ${ }^{11,12}$ It has been reported that mice fed with a high-fat diet (HFD) enriched in cocoa butter, cholesterol, and cholate, develop a pathology that encompasses the clinical features of NASH. ${ }^{13}$ Using this experimental in vivo model, we studied whether a pre-existent, diet-induced, steatohepatitis exacerbates the severity of psoriasiform dermatitis induced by topical application of imiquimod (IMQ), a Toll-like receptor 7 agonist previously described to induce psoriasis-like skin inflammation. ${ }^{14}$ The results presented herein show that the presence of a cutaneous inflammatory stage, characteristic of steatohepatitis, favors the development of severe cutaneous inflammatory disease.

\section{Materials and Methods}

\section{Animals and Treatment Protocol}

Male C57BL/6J mice were purchased at 8 to 12 weeks of age (Janvier Labs, le Genest-Saint-Isle, France). The animals were divided into two groups of diet: mice given a standard diet (SD) (5001, LabDiet, St. Louis, MO), and mice given a HFD enriched in cocoa butter, cholesterol, and cholate $(0.5 \%)$ (9G21, TestDiet, St. Louis, MO). This diet containing 85.6\% energy from fat (fat composition is $58.3 \%$ saturated fatty acids, $39.7 \%$ unsaturated fatty acids, and $2 \%$ cholesterol), $14.3 \%$ from carbohydrate, and $9.1 \%$ from protein, as described by Matsuzawa et al. ${ }^{13}$ Mice received food and water ad libitum. After 9 weeks of diet, the back skin of all animals was depilated (Veet cream; Reckitt Benckiser, Slough, UK) on a 6-cm² surface. Both SD- and HFD-fed mice received, every other day until week 11, either a topical application of $62 \mathrm{mg}$ of IMQcontaining cream (Aldara 5\% cream; Meda, Solna, Sweden) or control cream (vaseline) on the dorsal skin and right ear, or were untreated. The protocol was approved by the regional ethics committee for animal experimentation (COMETHEACE86). To further elicit exacerbated skin inflammation, SD mice received intradermal injections of IL-17A in back skin and right ear (250 ng on each site, in $20 \mu \mathrm{L}$ phosphate-buffered saline; Peprotech, Rocky Hill, NJ) every other day for 1 week, or a single injection of IL-1 $\beta$ in right ear $(250 \mathrm{ng}$, in $20 \mu \mathrm{L}$ phosphate-buffered saline; Peprotech). Control mice received the vehicle phosphate-buffered saline injection.

\section{Severity Score of Skin Inflammation}

Skin inflammation in mice was evaluated using a score adapted from the clinical psoriasis area and severity index. The cumulative score ( 0 to 12 ) was the sum of three criteria, erythema, scaling, and thickening, ranging from 0 to 4 with 0 , none; 1 , slight; 2 , moderate; 3 , marked; 4 , very marked.

\section{Histological Analysis}

Epidermal thickening was measured every $50 \mu \mathrm{m}$ for the entire length of four random sections. Liver inflammation was evaluated from the NAFLD activity score (NAS) that is used in human pathology. This semiquantitative score (0 to 8$)$ represents the sum of scores for steatosis $(0,<5 \% ; 1,5 \%$ to $33 \% ; 2$, $34 \%$ to $66 \% ; 3,>66 \%$ ), lobular inflammation was evaluated at a $\times 200$ magnification ( 0 , no foci; $1,<2$ foci; 2,2 to 4 foci; 3 , $>4$ foci) and hepatocyte ballooning ( 0 , none; 1 , few ballooned cells; 2, prominent ballooning). All slides were blindly scored (P.V. and L.S.).

\section{Immunofluorescence}

Frozen sections of back skin ( $8 \mu \mathrm{m}$ thick) were fixed in acetone/ methanol and stained with a rat anti-CD45 mAb (clone 30F11, 1:200; BD Biosciences, Franklin Lakes, NJ). After washing, the slides were incubated for 60 minutes at room temperature with a donkey-anti-rat fluorescein isothiocyanate $\mathrm{Ab}$ (1:200; Jackson ImmunoResearch, West Grove, PA). Nuclei were stained with To-Pro3 (1:800; Life Technologies, Carlsbad, CA). Confocal microscopy was performed using an Olympus FV1000 microscope (ImageUP microscopy platform; University of Poitiers). Quantification of cutaneous $\mathrm{CD} 45^{+}$cells per high-power field $(\times 400$ magnification) was made from four randomly chosen high-power fields per mouse ( $n=5$ per group).

\section{Detection of Active Caspase-1}

The presence of active caspase-1 in back skin sections was detected with the fluorescent-labeled caspase-1 inhibitor FAM-FLICA (1:50), according to the manufacturer's instructions (Immunochemistry Technologies, Bloomington, $\mathrm{MN}$ ). Nuclei were stained with To-Pro3. Analysis was performed with confocal microscopy.

\section{Cell Preparation and Flow Cytometry}

Lymph node cell suspensions were obtained after mechanical disruption and were filtered through a $100-\mu \mathrm{m}$ mesh. Skin was treated with Mouse Epidermis Dissociation Kit (Miltenyi Biotec, Bergisch Gladbach, Germany), and epidermal cell suspensions were obtained according to the manufacturer's instructions. After disruption, dermis was incubated in 80 $\mu \mathrm{g} / \mathrm{mL}$ Liberase TM Research Grade (Roche Diagnostics, Basel, Switzerland) and $10 \mu \mathrm{g} / \mathrm{mL}$ DNase I solution (SigmaAldrich, St. Louis, MO) for 1 hour at $37^{\circ} \mathrm{C}$ before shredding with gentle MacsDissociator (Miltenyi Biotec) and finally filtered through a $40-\mu \mathrm{m}$ mesh. After gathering dermal and epidermal suspensions, cells were incubated for 4 hours in the presence of Golgi Plug ( $1 \mu \mathrm{L} / 10^{6}$ cells; BD Biosciences), with or without $50 \mathrm{ng} / \mathrm{mL}$ phorbol myristate acetate and $750 \mathrm{ng} / \mathrm{mL}$ 
ionomycin. Cells were incubated with Fc Block (BD Biosciences) for 10 minutes and then for 15 minutes at $4{ }^{\circ} \mathrm{C}$ with the following antibodies: V500-conjugated anti-CD45, BV421-conjugated anti-CD3e, PECy7-conjugated anti-CD4, fluorescein isothiocyanate-conjugated anti-TCR $\gamma \delta$ (all from BD Biosciences) and Zombie NIR (BioLegend, San Diego, CA). For intracellular staining, cells were permeabilized with Cytofix/Cytoperm and labeled with PE-conjugated anti-IL17A. IgG1, $\kappa$ was used as isotype control (BD Biosciences). Data were collected with a FACSVerse instrument and analyzed using FACSuite software version 1.0 (BD Biosciences).

\section{Quantitative RT-PCR Analysis}

Skin and liver total RNA were isolated using NucleoSpin RNA II kit (Macherey-Nagel, Hoerdt, France) and reverse- transcribed with SuperScript II Reverse Transcriptase (Life Technologies) according to the manufacturer's instructions. Quantitative RT-PCR was performed using the LightCycler-FastStart DNA Master ${ }^{\text {Plus }}$ SYBRGreen I kit on LightCycler 480 (Roche Diagnostics). Sense and antisense oligonucleotides were purchased from Eurogentec (Angers, France).

\section{Insulin Tolerance Test}

At week 9, mice were injected i.p. with $0.5 \mathrm{U} / \mathrm{kg}$ of body weight insulin (Humalog; Lilly, Indianapolis, IN) following a 4-hour fast. Glucose values were measured with a blood glucose monitoring system (FreeStyle Easy; Abbott, Chicago, IL) 0, 30, 60, 90, and 120 minutes after insulin injection.
A

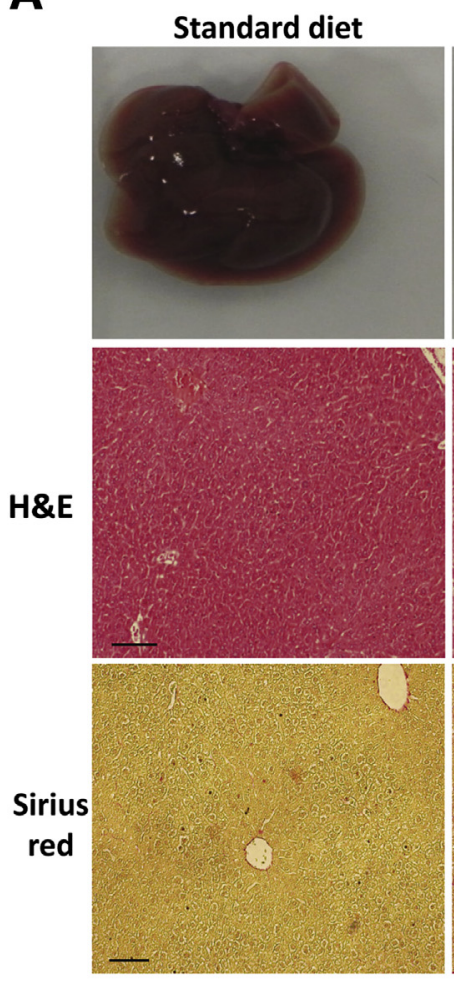

\section{High-fat diet}
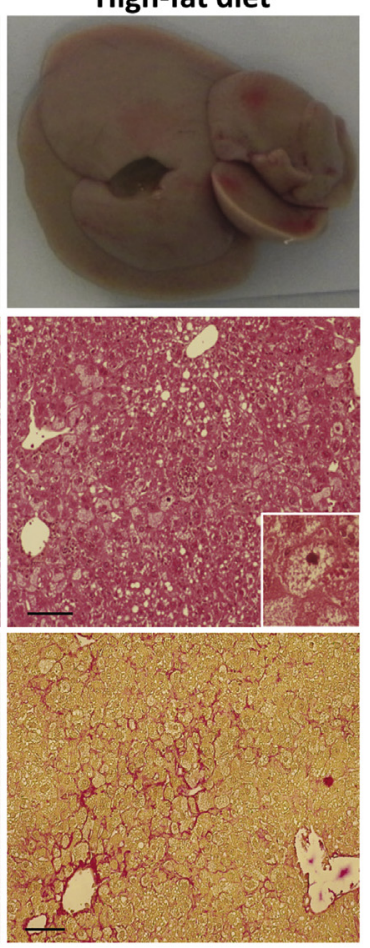

B
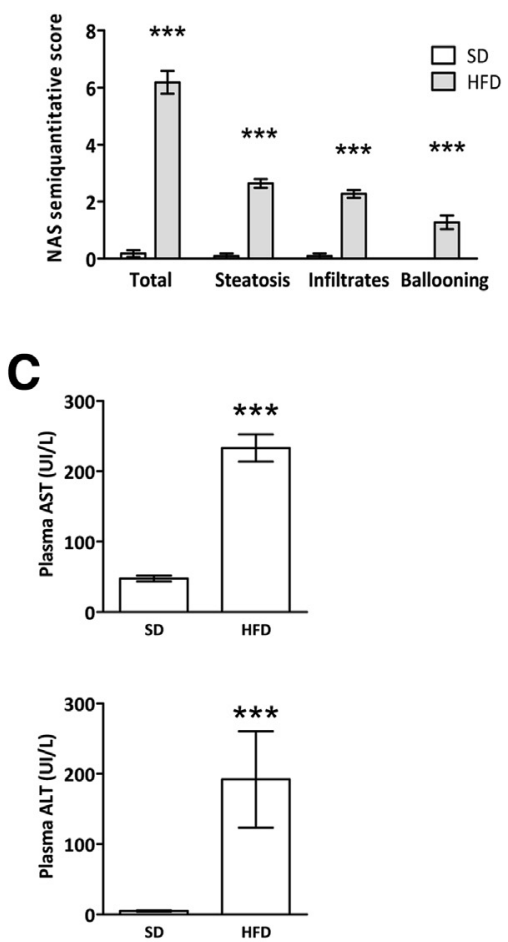

D
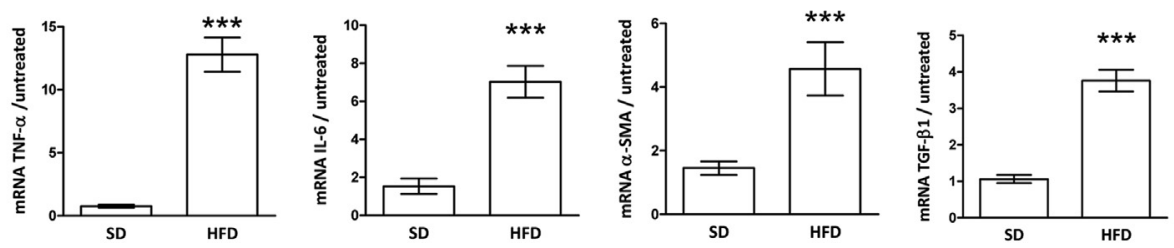

Figure 1 High-fat diet (HFD) induces steatohepatitis. Mice were fed a HFD or a standard diet (SD) for 11 weeks. A: Aspect of livers and representative histology stained with hematoxylin and eosin (H\&E) and Sirius red. Ballooning hepatocyte is shown in the inset. B: Nonalcoholic fatty liver disease activity semiquantitative score. C: Levels of transaminases in plasma. D: Liver mRNA relative expression of tumor necrosis factor (TNF)- $\alpha$, IL-6, $\alpha$-smooth muscle actin ( $\alpha$ SMA), and transforming growth factor (TGF)- $\beta 1$ normalized to glyceraldehyde-3-phosphate dehydrogenase and expressed as fold increase compared to untreated mice. ${ }^{* *} P<0.001$. Scale bar $=100 \mu \mathrm{m}(\mathbf{A})$. Original magnification, $\times 100(\mathbf{A})$. ALT, alanine aminotransferase; AST, aspartate aminotransferase. 


\section{Biochemistry}

Triglycerides, alanine aminotransferase, aspartate aminotransferase, and glucose were measured with a clinical calibrated analyzer (Cobas; Roche Diagnostics).

\section{Statistical Analysis}

Results are presented as means \pm SEM of four to eight mice per group from four independent experiments. Nonparametric Kruskal-Wallis with a Dunns post-test or Mann-Whitney test were used for the statistical evaluation (Prism software version 5.01; GraphPad Software, San Diego, CA). $P<0.05$ was considered significant.

\section{Results}

\section{HFD Induces Steatohepatitis}

Mice fed with a HFD enriched in cocoa butter, cholesterol, and cholate displayed hepatomegaly, as compared to control standard diet (SD)-fed mice, and their livers were pale. Histological analysis revealed hepatocyte ballooning, an important diagnostic feature of human NASH, as well as major microvacuolar and macrovacuolar steatosis. Lobules were infiltrated with mononuclear cells and polymorphonuclear neutrophils (Figure 1A). The mean histological activity score, which is $\geq 5$ for most patients with definite NASH, ${ }^{15}$ was $6.2 \pm 0.3$ (Figure 1B). Hepatocellular injury in the HFD group was reflected by elevated serum levels of aspartate aminotransferase and alanine aminotransferase (Figure 1C). Expressions of tumor necrosis factor- $\alpha$ and IL- 6 transcripts were strongly enhanced in the livers of HFD-fed animals, reflecting ongoing inflammation in this organ. HFD induced an overexpression of $\alpha$-smooth muscle actin and transforming growth factor- $\beta$, two markers of activated hepatic stellate cells (Figure 1D), which is consistent with the general notion that these cells are the major source of liver fibrosis. Accordingly, Sirius red staining revealed chicken-wired fibrosis in perisinusoidal areas in the livers of HFD-fed mice, as well as periportal collagen deposition (Figure 1A).

\section{HFD Does Not Induce Systemic Insulin Resistance}

HFD-fed mice were not obese and did not show expansion of adipose tissue. Moreover, their weight gain was lower as compared to that of control mice. Similarly, plasma triglycerides and fasting blood glucose were not increased in the HFD group. To evaluate systemic insulin sensitivity, an insulin tolerance test was conducted. The decrease in blood glucose after insulin injection was similar in both diets, indicating that HFD mice did not exhibit systemic insulin resistance (Table 1).

\section{Psoriasiform Dermatitis Is Aggravated in Mice with Steatohepatitis}

Starting 9 weeks after the onset of the food regimen, both HFD- and SD-fed mice were treated every other day for 14 days with Aldara containing 5\% IMQ. Control mice, fed with a SD, received a vaseline application (SD-VAS) for the same period of time. Aldara treatment of SD mice (SD-IMQ) resulted in a moderately erythematous and squamous skin showing macroscopic features of psoriasis that peaked at day 7. Thereafter, inflammatory signs receded and the skin showed only few scales at day 14 (Figure 2, A and B). Histological analysis revealed psoriasiform changes with foci of parakeratosis, indicating incomplete maturation of keratinocytes, an acanthosis, a hyperkeratosis, and cellular dermal infiltrates (Figure 2A). Aldara induced more severe macroscopic signs of inflammation, starting from day 4 onward, in HFD-IMQ mice, as compared to SD-IMQ mice. At day 7, a distinct erythema with a marked thickening of the dorsal skin was observed and at day 14, scales were conspicuously present, reminiscent of human psoriasis (Figure 2, A and B). Moreover, epidermis hyperplasia was more prevalent in HFD-IMQ mice than in SD-IMQ mice $(90.1 \pm 3.5$ versus $62.2 \pm 2.7 \mu \mathrm{m} ; P=0.0002$ ) (Figure 2, A and C), although both groups presented the same psoriasiform features, as shown by hematoxylin and eosin coloration of the back skin (Figure 2A). Similarly, overexpression of inflammatory markers of psoriasis was exacerbated in HFD-IMQ mice. At

Table 1 High-Fat Diet Does Not Induce Features of the Metabolic Syndrome

\begin{tabular}{|c|c|c|c|}
\hline Feature & Standard diet & High-fat diet & $P$ value \\
\hline Body weight increase (\%) & $121 \pm 2.1$ & $101 \pm 3.5$ & 0.0002 \\
\hline Epidydimal fat pad weight (g) & $0.18 \pm 0.06$ & $0.16 \pm 0.06$ & 0.79 \\
\hline Plasma triglycerides $(\mathrm{g} / \mathrm{L})$ & $0.45 \pm 0.03$ & $0.32 \pm 0.04$ & 0.002 \\
\hline Fasting blood glucose $(\mathrm{g} / \mathrm{L})$ & $1.09 \pm 0.06$ & $0.93 \pm 0.04$ & 0.04 \\
\hline \multicolumn{4}{|l|}{ Insulin tolerance test* } \\
\hline$\%$ Initial blood glucose 30 minutes & $63.2 \pm 1.4$ & $62.5 \pm 5.3$ & 1 \\
\hline$\%$ Initial blood glucose 120 minutes & $94.6 \pm 1.7$ & $92.6 \pm 7.4$ & 1 \\
\hline
\end{tabular}

Data are presented as means \pm SEM.

*Insulin tolerance test was conducted at week 9. 
A

SD-VAS
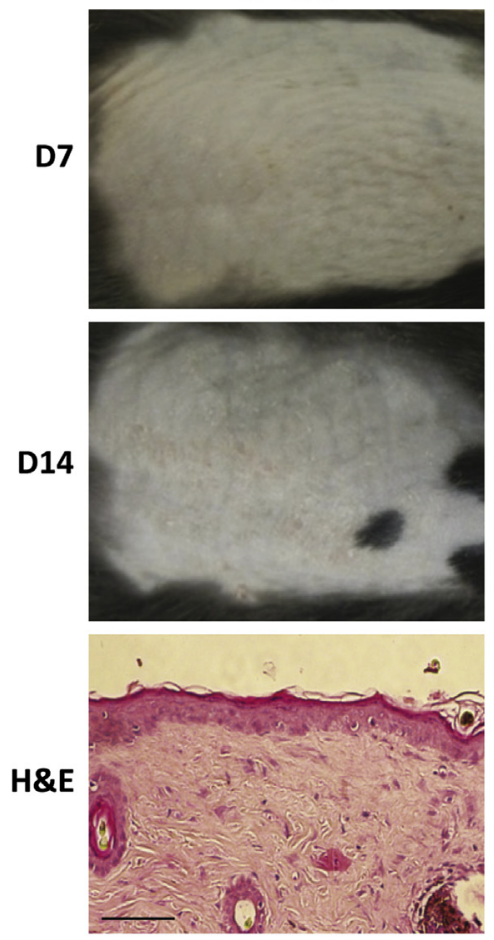

B

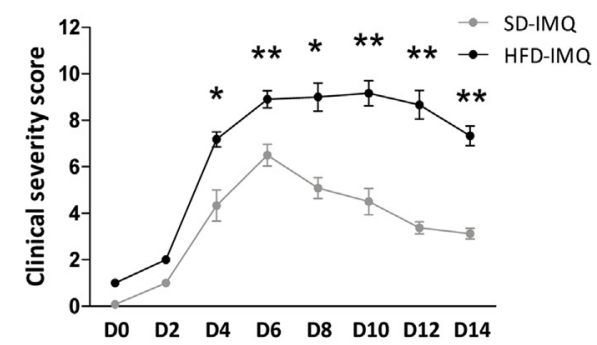

D

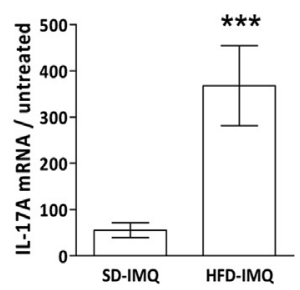

SD-IMQ
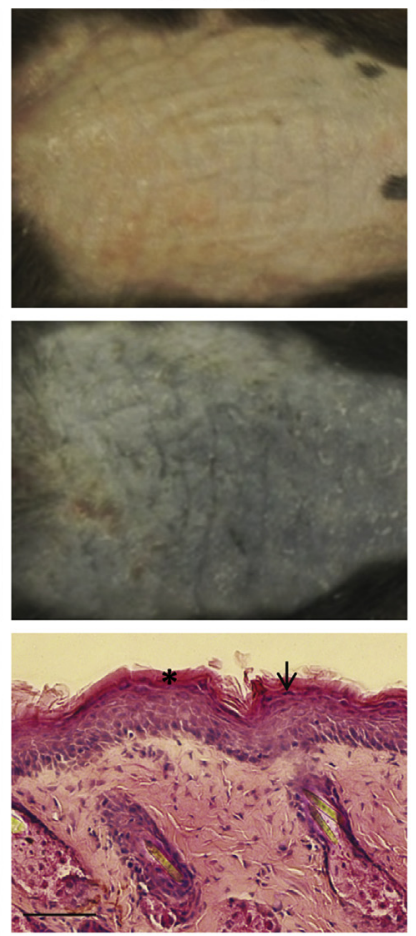

HFD-IMQ
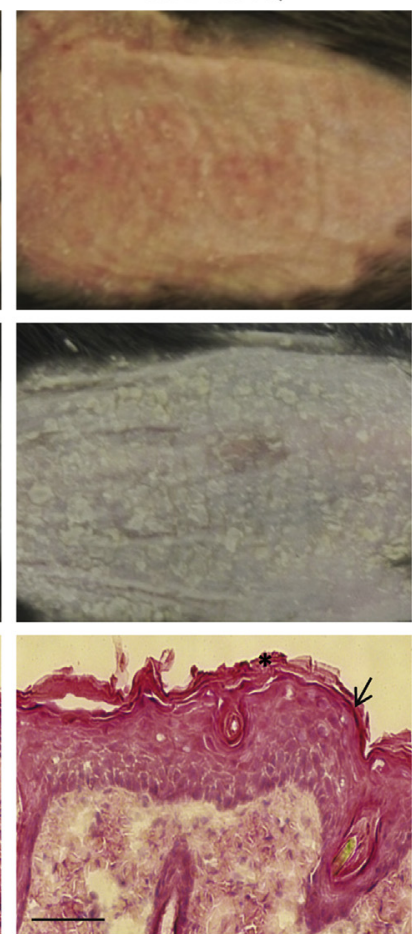

C

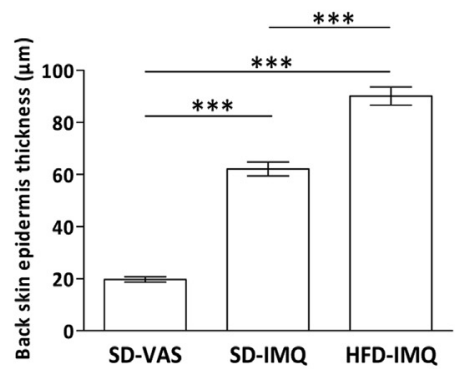

Figure 2 Mice fed the high-fat diet (HFD) display aggravated psoriasiform dermatitis. After 9 weeks, the mice received 2 weeks of topical treatment of imiquimod (IMQ)-containing cream or control vaseline (VAS). A: Standard diet (SD) and HFD mice after topical treatment, and representative hematoxylin and eosin (H\&E)-stained sections of dorsal skin at day 14. Arrows indicate parakeratosis, asterisks indicate hyperkeratosis. B: Clinical severity score. C: Epidermal thickening in IMQ-treated mice. D: Back skin mRNA relative expression at day 7 of IL-17A, IL-22, S100A9, and CXCL3 normalized to glyceraldehyde-3phosphate dehydrogenase and expressed as fold increase compared to untreated mice. ${ }^{*} P<0.05,{ }^{* *} P<0.01$, and ${ }^{* * *} P<0.001$. Scale bar $=50 \mu \mathrm{m}$ (A). Original magnification, $\times 100($ A).

day 7, expression levels of IL-17A and IL-22 were approximately sevenfold higher in the skin of HFD-IMQ mice, as compared to SD-IMQ mice $(P=0.0005$ and $P=0.0019$, respectively), as were those of S100A9 and the neutrophil-attracting chemokine CXCL3 $(P<0.0001$ and $P=0.004$, respectively) (Figure 2D).

\section{Steatohepatitis Is Associated with a Cutaneous Production of IL-17A}

Because the IMQ-induced dermatitis was more severe in HFD-fed mice, we wondered whether the exacerbation was linked to a latent cutaneous proinflammatory state in 
A
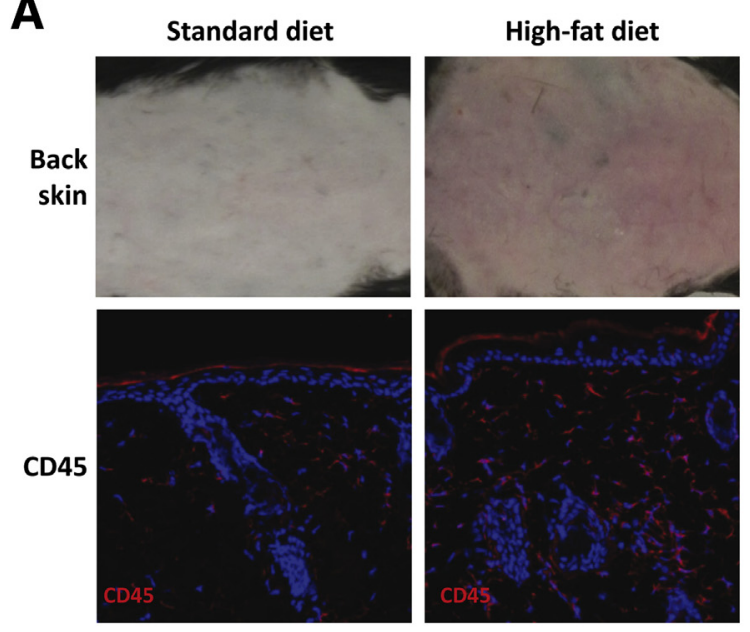

C

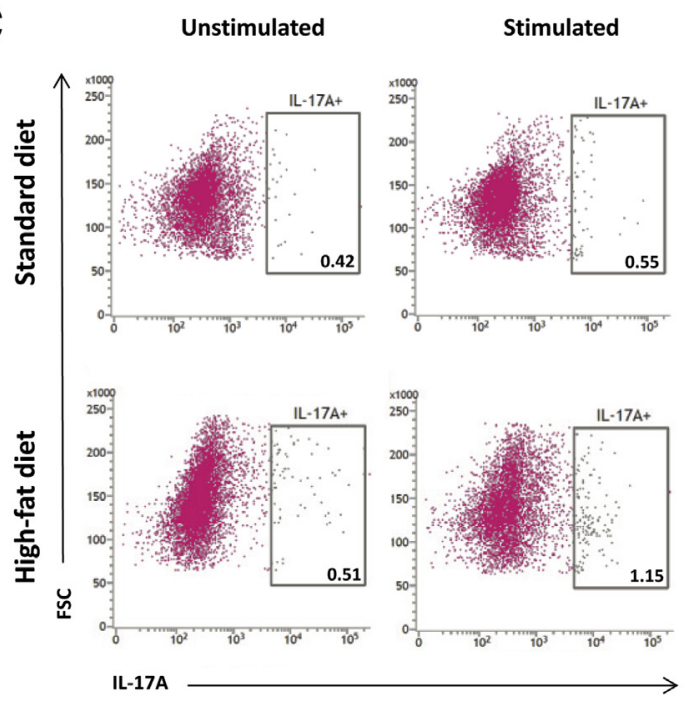

B
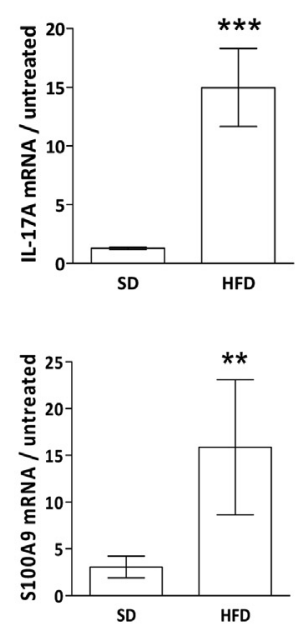

D

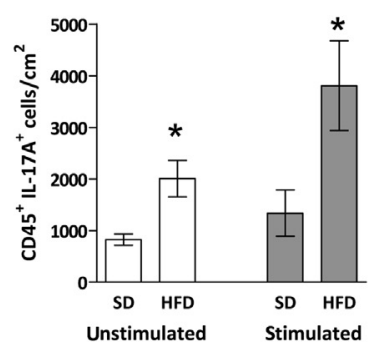

Figure 3 High-fat diet (HFD) induces an inflammatory state of the skin with local production of IL-17A. A: Mouse depilated skin after 9 weeks of diet and representative CD45 immunostaining of skin sections. B: Skin mRNA relative expression of IL-17A and S100A9 normalized to glyceraldehyde3-phosphate dehydrogenase and expressed as fold increase compared to untreated mice. C: Flow cytometry analysis of IL-17A ${ }^{+}$cells collected from the dorsal skin, unstimulated or stimulated for 4 hours with phorbol myristate acetate and ionomycin. Cells were gated using a viability marker (Zombie NIR) then on the $\mathrm{CD}_{4} 5^{+}$population. Percentages shown in the gate represent the mean percentage obtained from three to five mice in each group from two independent experiments. D: Absolute quantification of IL-17A ${ }^{+}$leukocytes in dorsal skin. ${ }^{*} P<0.05,{ }^{* *} P<0.01$, and $* * * P<0.001$. Original magnification, $\times 400($ A). SD, standard diet. these animals. Indeed, a discrete ongoing erythema was present in untreated HFD-fed mice (Figure 3A). Analysis of hematoxylin and eosin-stained sections of dorsal skin did not reveal any change in the structure of the epidermal layer of HFD-fed mice, whereas an inflammatory infiltrate was detected in the dermis when compared to SD-fed mice. Immunostaining showed increased infiltration of $\mathrm{CD} 45^{+}$cells in the dorsal skin of HFD-fed mice when compared to SD-fed mice (33.6 \pm 3.2 versus $23.1 \pm 2$ stained cells per high-power field, $P=0.03$ ), that preferentially localized in the dermis (Figure $3 \mathrm{~A}$ ). We further addressed whether the exacerbation of the psoriasiform dermatitis noted in HFD-fed mice could be linked to the presence of proinflammatory cytokines known to be involved in the dermatitis and produced by $\mathrm{CD} 45^{+}$leukocytes. Indeed, IL-17A was expressed in both the back skin (Figure 3B) and the ear skin (data not shown) of HFD-fed mice in contrast to SD-fed mice. We did not detect any cutaneous overexpression of the Th17-related cytokines IL-23, IL-22, IL-17C, or IL-17F (data not shown). Interestingly, S100A9, known to be produced by keratinocytes in response to IL-17A, was overexpressed in the skin of HFD-fed mice (Figure 3B). By using flow cytometry analysis, we observed an increased proportion of IL-17A $\mathrm{A}^{+}$leukocytes in the back skin of HFD-fed mice as compared to control mice $(0.51 \%$ versus $0.42 \%$ of $\mathrm{CD}^{+} 5^{+}$cells; $P=0.03$ ) (Figure $3 \mathrm{C}$ ), as well as their absolute number (Figure 3D). The differences in proportion and number of cutaneous $\mathrm{IL}-17 \mathrm{~A}^{+}$leukocytes between the HFD and SD regimen were further exacerbated after stimulation of the cell suspensions with phorbol myristate acetate and ionomycin (Figure 3, C and D).

\section{IL-17A Mediates the Exacerbation of Psoriasiform Dermatitis in Mice with Steatohepatitis}

To determine whether the increased presence of IL-17A in the skin of HFD-fed mice could account for the exacerbation of the experimentally induced psoriasiform dermatitis (induced thereafter), SD-fed mice received intradermal injections of rIL-17A, concomitantly with the topical application of IMQ, or vaseline as a control, every other day for 1 


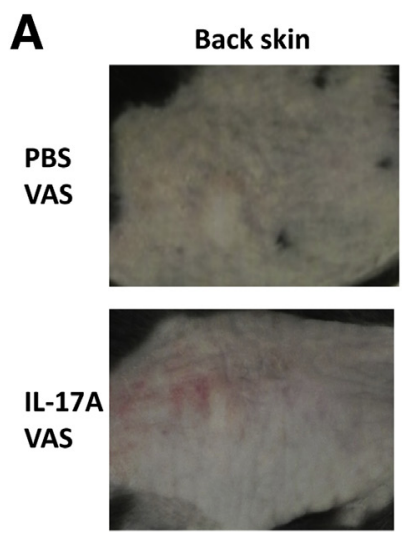

C

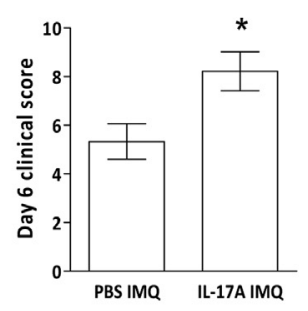

E

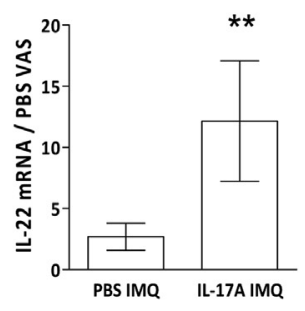

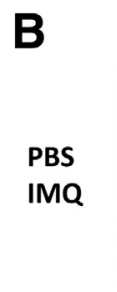
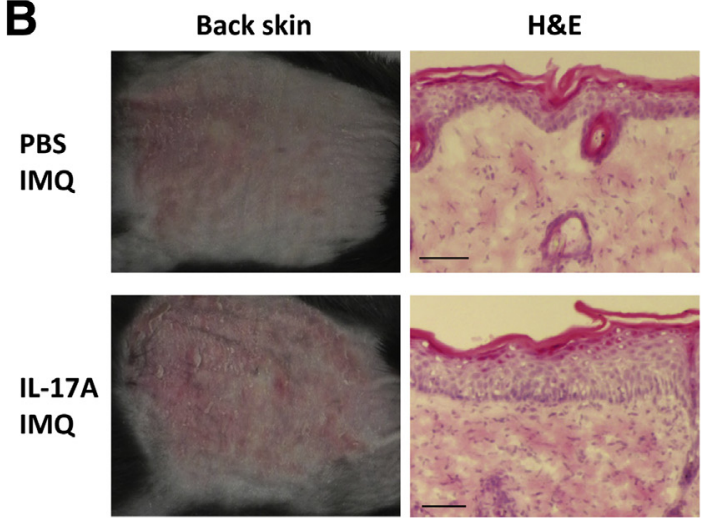

D
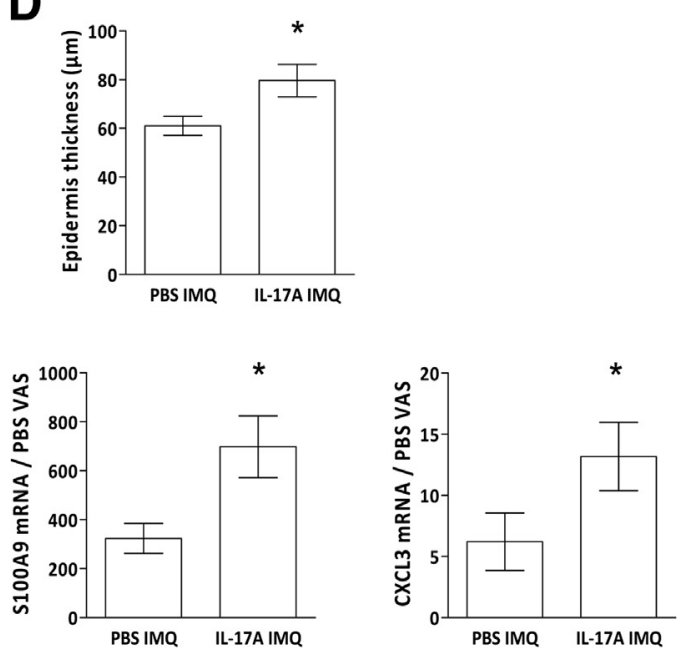

Figure 4 Intradermal injection of IL-17A recapitulates features of skin inflammation observed in high-fat diet (HFD) mice. Mice fed with a standard diet (SD) received an intradermal injection of rIL-17A or vehicle every other day for 1 week, as well as a topical treatment with imiquimod (IMQ) or vaseline (VAS). A: VAS-treated mice at day 7, receiving either rIl-17A (IL-17A VAS) or the vehicle [phosphate-buffered saline (PBS) VAS]. B: IMQ-treated mice at day 7 , receiving either rIl17A (IL-17A IMQ) or the vehicle (PBS IMQ). C: Cumulative clinical score of skin inflammation. D: Histological measurement of epidermal thickening. E: Dorsal skin mRNA relative expressions at day 7 of IL-17A, S100A9, IL-22, and CXCL3 normalized to glyceraldehyde-3-phosphate dehydrogenase and expressed as fold increase compared to PBS VAS mice. ${ }^{*} P<0.05,{ }^{* *} P<0.01$. Scale bar $=100 \mu \mathrm{m}$ (B). H\&E, hematoxylin and eosin. week. Mice receiving rIL-17A and treated with vaseline showed a skin erythema similar to that of HFD-fed mice before the induction of dermatitis with IMQ (Figure 4A). Mice treated with rIL-17A and IMQ (IL-17A IMQ) developed a severe dermatitis with pronounced erythema and skin thickening (Figure 4, B and C), as well as exacerbated acanthosis (79.6 \pm 6.7 versus $61 \pm 3.9 \mu \mathrm{m} ; P=0.03)$ (Figure 4, B and D), to the same extent as that of HFD-fed mice treated with IMQ. Furthermore, IMQ-induced expression of IL-22, S100A9, and CXCL3 transcripts in the dorsal skin was strongly enhanced in mice that also received rIL-17A (Figure $4 \mathrm{E}$ ).

\section{Steatohepatitis Is Associated with an Expansion of Th17 Cells in Lymph Nodes}

To further determine the cellular sources of IL-17A in HFDfed mice before the induction of psoriasiform dermatitis, the phenotype of $\mathrm{IL}-17 \mathrm{~A}^{+}$cells obtained from the draining lymph nodes was analyzed. Under unstimulated condition, untreated HFD-fed mice showed an expansion of CD45 IL-17A ${ }^{+}$leukocytes as compared to untreated SD-fed mice, most of them expressing CD3 (Figure 5, A and C). The proportion and absolute number of $\mathrm{CD}^{+} \mathrm{CD}^{+} \mathrm{TCR} \gamma \delta^{-}$ Th17 lymphocytes were increased in the untreated HFD-fed mice, as compared to the untreated SD-fed animals (Figure 5, A and C). After stimulation with phorbol myristate acetate and ionomycin, the proportions and absolute number of $\mathrm{IL}_{-17 \mathrm{~A}^{+}} \mathrm{CD}^{+} \mathrm{CD}^{+}$Th17 lymphocytes increased in untreated HFD-fed mice, whereas the percentages of $\mathrm{IL}-17 \mathrm{~A}^{+} \mathrm{CD}^{+} \mathrm{TCR} \gamma \delta^{+}$cells were similar in both diets (Figure 5, B and $\mathrm{C}$ ).

\section{Mice with Steatohepatitis Display Epidermal Activation} of Caspase-1

It is known that Th17 lymphocytes are trafficking into inflamed tissues, including skin, in part as a result of the interaction between CCR6 and its specific ligand CCL20. Indeed, CCL20 transcripts were overexpressed in the skin of HFD-fed mice (Figure 6A). Proinflammatory cytokines and in particular IL-1 $\beta$ have been reported to induce the cutaneous production of CCL20. ${ }^{7,16}$ Indeed, pro-IL-1 $\beta$ was also overexpressed in the skin of HFD-fed mice (Figure 6A). This prompted us to study cutaneous caspase-1, the inflammasome-activated enzyme required to process the active form of IL-1 $\beta$. By using an inhibitory peptide coupled to a fluorescent tag that binds to the reactive center of activated caspase-1, we detected spots of intracytoplasmic caspase-1 labeling in the epidermis of 

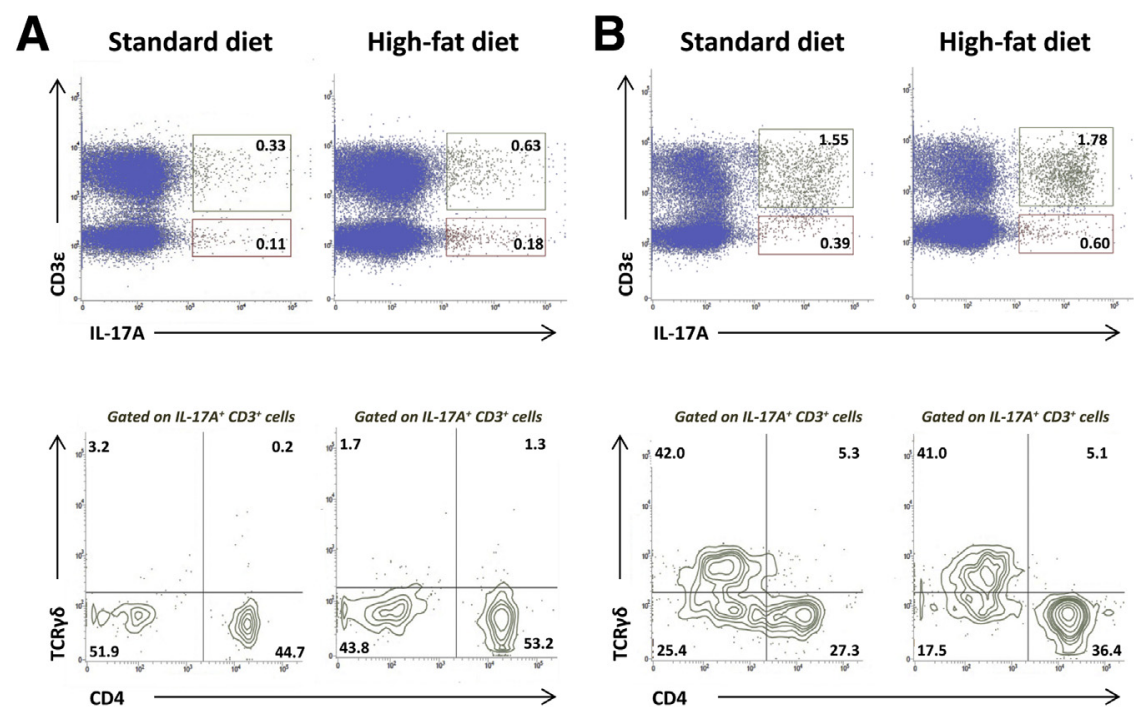

C
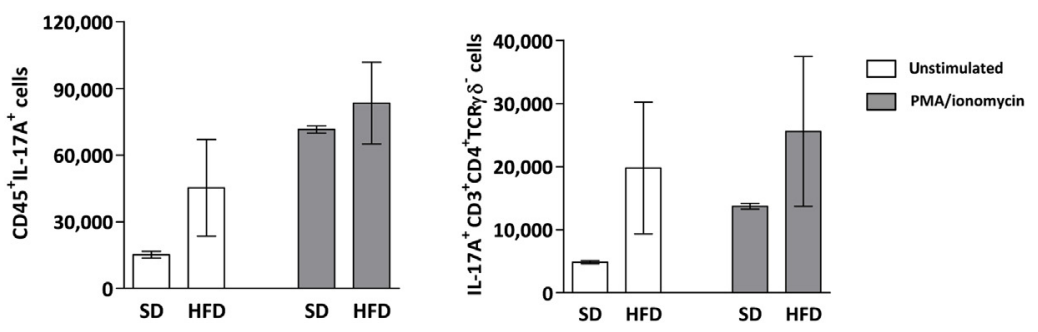

Figure 5 High-fat diet (HFD) promotes the accumulation of $\mathrm{T}$ helper 17 lymphocytes in the draining lymph nodes of untreated mice. Flow cytometry analysis of single-cell suspensions obtained from draining lymph nodes without $(\mathbf{A})$ or with phorbol myristate acetate (PMA) and ionomycin stimulation (B). Live cells were gated using a viability marker (Zombie NIR) then on the $\mathrm{CD} 45^{+}$ population. Cells were further gated on IL-17A ${ }^{+}$ $\mathrm{CD}^{+}$populations. Mean percentages are indicated in each quadrant. C: Absolute quantification of $\mathrm{CD}_{4} 5^{+} \mathrm{IL}^{-17 \mathrm{~A}^{+}}$leukocytes and of $\mathrm{CD} 3^{+} \mathrm{CD}^{+}{ }^{+} \mathrm{TCR} \gamma \delta^{-}$ Th17 lymphocytes in the draining lymph nodes, without or with PMA and ionomycin stimulation. Data represent the means from two to four mice per group from one experiment. mice fed with the HFD, but not in SD-fed mice (Figure 6B). To confirm the putative in vivo role of IL-1 $\beta$, SD-fed mice received intradermal injection of rIL-1 $\beta$. A single injection induced an early cutaneous expression of CCL20 mRNA, as observed in HFD-fed mice, and subsequently an overexpression of IL-17A transcripts at day 3 (Figure 6C).

\section{Discussion}

The growing number of studies reporting an increase in the prevalence of NAFLD in psoriatic patients ${ }^{2,4,17,18}$ led us to experimentally address the consequences of this systemic disorder on the development and severity of psoriasis. We used the murine model of psoriasiform dermatitis induced by topical application of IMQ-containing cream. In addition to the macroscopic and histological skin aspects that closely resemble the human disease, the pathogenesis of IMQinduced dermatitis shares key molecular pathways with psoriasis, including its dependence on the activity of $\mathrm{T}$ lymphocytes and the implication of the IL-23/17 axis. ${ }^{19}$ The results from the present study show that a pre-existing condition of a diet-induced steatohepatitis facilitates the inflammatory process leading to aggravated psoriasiform dermatitis. This exacerbation was characterized by excessive scale formation, increased epidermal hyperplasia, and increased overexpression of S100A9 and CXCL3, known to be produced by activated keratinocytes. Moreover, IL-17A and IL-22, cytokines that play a pivotal role in the initiation of dermatitis, ${ }^{14,20}$ were strongly overexpressed in these animals. Our findings are in agreement with recent clinical studies reporting that presence of NAFLD is associated with more severe skin lesions. ${ }^{17,18,21}$ Notably, the study of Roberts et $\mathrm{al}^{18}$ revealed an increasing trend in the severity of psoriasis when patients progressed from steatosis to NASH. In addition to the deleterious role of obesity on psoriasis, ${ }^{22}$ this observation suggests a specific role of liver inflammation on psoriatic disease. To focus on the liver itself, we then chose a HFD model of NASH where the addition of cholesterol and cholate does not induce obesity or features of metabolic syndrome. More important, this diet induces key features of human NASH represented by ballooned hepatocytes, lipid-induced oxidative stress, and hepatic insulin resistance. ${ }^{13,23}$

Our experiments indicate that HFD-induced IL-17A aggravates psoriasiform dermatitis in NASH mice, corroborating its key role in the development of psoriasis and IMQ-induced dermatitis. ${ }^{24-26}$ Intradermal injection of rIL-17A in SDfed animals, recapitulated steatohepatitis-associated skin erythema, and subsequent IMQ treatment resulted in comparable exacerbation of dermatitis as that of mice with steatohepatitis (ie, aggravated scale formation, acanthosis, and overexpression of IL-22, S100A9, and CXCL3 in the affected skin). Local production of IL-17 in the lung or joints has been linked to the exacerbation of secondary experimental airway 
A
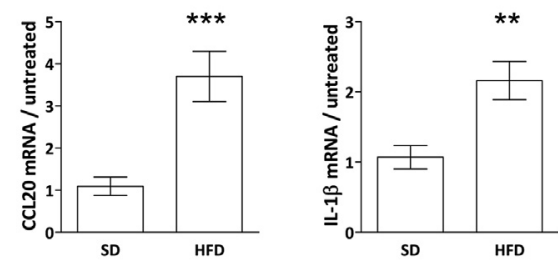

B
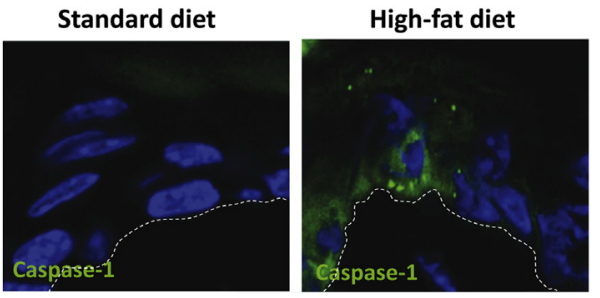

C
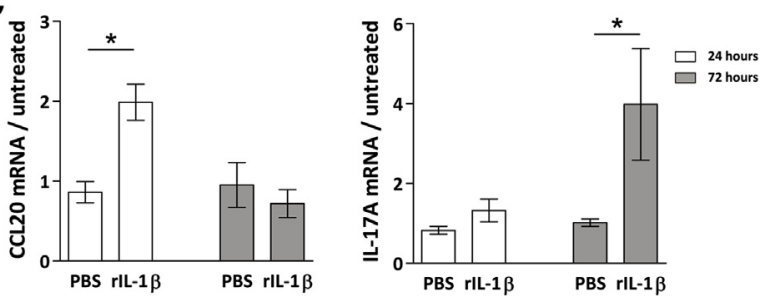

Figure 6 The caspase-1/IL-1 pathway is induced in the skin of untreated mice with steatohepatitis. A: Dorsal skin mRNA relative expressions of chemokine ligand (CCL) 20 and IL-1 $\beta$ in standard diet (SD) and high-fat diet (HFD) mice, normalized to glyceraldehyde-3-phosphate dehydrogenase (GAPDH) and expressed as fold increase compared to untreated mice. B: Caspase-1 activity was detected from dorsal skin sections of HFD and SD mice. Dotted lines indicate dermoepidermal junctions. C: CCL20 and IL-17A cutaneous expression 24 and 72 hours after a single intradermal injection of IL-1 $\beta$ in the ear of SD-fed mice. Results are normalized to GAPDH and expressed as fold increase compared to untreated mice. ${ }^{*} P<0.05$, $* * P<0.01$, and ${ }^{* * *} P<0.001$. Original magnification, $\times 600$ (B).

responsiveness or collagen-induced arthritis in mice fed with a HFD, and HFD-associated bronchial hyperractivity was totally abolished in $I L-17 A^{-/-}$mice. . $^{27,28}$

HFD-fed mice had a higher proportion of cutaneous IL-17A-producing leukocytes. As their restricted number in the dorsal skin limited the exploration of the cellular sources, we studied the draining lymph nodes. Among the $\mathrm{IL}-17 \mathrm{~A}^{+}$cells, the number and proportion of Th17 lymphocytes was increased in mice with steatohepatitis, which was consistent with a recent report showing that a long-term high-fat regimen induced skin lesions with cutaneous overexpression of IL-17A, and a higher proportion of CD4 $4^{+}$ IL-17A ${ }^{+}$cells in the draining lymph nodes. ${ }^{29}$ The expansion of Th17 lymphocytes in the draining lymph nodes of mice with steatohepatitis, along with the cutaneous overexpression of CCL20, argues for a role of CCR6 and its ligand in the specific migration of these cells into the inflamed skin before the induction of psoriasiform dermatitis. As IL- $1 \beta$ is implicated in the epithelial production of IL-17A in models of high-fat feeding, ${ }^{29,30}$ and can induce the synthesis of CCL20 by keratinocytes, ${ }^{7,31}$ we studied its role in the cutaneous inflammation observed in HFD-fed mice. IL-1 $\beta$ was indeed overexpressed in the skin of mice with steatohepatitis, and its processing enzyme caspase-1 was activated in the epidermal layer. Finally, intradermal injection of rIL-1 $\beta$ induced cutaneous expression of CCL20, before that of IL-17A, suggesting that the activation of caspase-1 in the epidermis of HFD-fed mice is an upstream event of maturation of IL-1 $\beta$ and, subsequently, production of IL-17A. Like caspase-1, the nucleotide-binding domain, leucine-rich repeats containing family, pyrin domaincontaining-3 (NLRP3) inflammasome has also been implicated in the production of IL-17A, as demonstrated in the lungs of mice fed with a HFD. ${ }^{28}$ Caspase- 1 is activated through several inflammasomes, ${ }^{32}$ although the signaling cascade leading to its activation in our experimental model remains to be determined. From our results, we cannot state whether skin inflammation observed in HFD-fed mice is directly or indirectly induced by steatohepatitis. Nevertheless, because caspase-1 and NLRP3 colocalize in keratinocytes cultured with high concentrations of lipids, ${ }^{29}$ we hypothesize that the increase in circulating very-low-density lipoprotein-cholesterol and low-density lipoprotein-cholesterol, that are associated with steatohepatitis in our model, ${ }^{13}$ might activate the caspase-1/NLRP3 complex in the skin of HFD-diet mice.

In conclusion, we demonstrate that pre-existent steatohepatitis may lead to an exacerbation of psoriasiform dermatitis and that local, cutaneous, production of IL-17A is implicated in this process. This observation strengthens the notion of NAFLD as a disease with systemic manifestations, including skin inflammation. Psoriatic patients should be screened systematically for NAFLD, as the liver disease is likely to aggravate skin lesions, which could tentatively explain the resistance to biotherapy in some patients.

\section{Acknowledgments}

We thank Dr. Anne Cantereau for technical assistance in confocal microscopy (ImageUP, University of Poitiers) and Drs. Frédéric Favreau and Pierre Dupuis for routine biochemistry measurements (Poitiers University Hospital).

P.V. conceived experiments, acquired, analyzed, and interpreted data, and wrote and revised the manuscript; L.S. acquired and analyzed data; J.-F.J. analyzed and interpreted data and revised the manuscript; M.P. and I.P.-P. acquired data; A.D. analyzed data; P.L. analyzed and interpreted data; L.F. analyzed data and revised the manuscript; M.S. interpreted data; H.Y. interpreted data and revised the manuscript; F.M. analyzed and interpreted data, and revised the manuscript; and C.S. and J.-C.L. conceived experiments and interpreted data, and revised manuscript. P.V. is the guarantor of this work and, as such, had full access to all of the data in the study and takes responsibility for the integrity of the data and the accuracy of the data analysis. 


\section{References}

1. Armstrong MJ, Adams LA, Canbay A, Syn WK: Extrahepatic complications of nonalcoholic fatty liver disease. Hepatology 2014, 59: $1174-1197$

2. Van der Voort EA, Koehler EM, Dowlatshahi EA, Hofman A, Stricker BH, Janssen HL, Schouten JN, Nijsten T: Psoriasis is independently associated with nonalcoholic fatty liver disease in patients 55 years old or older: results from a population-based study. J Am Acad Dermatol 2014, 70:517-524

3. Miele L, Vallone S, Cefalo C, La Torre G, Di Stasi C, Vecchio FM, D’Agostino M, Gabrieli ML, Vero V, Biolato M, Pompili M, Gasbarrini G, Rapaccini G, Amerio P, De Simone C, Grieco A: Prevalence, characteristics and severity of non-alcoholic fatty liver disease in patients with chronic plaque psoriasis. J Hepatol 2009, 51: 778-786

4. Madanagobalane S, Anandan S: The increased prevalence of nonalcoholic fatty liver disease in psoriatic patients: a study from South India. Australas J Dermatol 2012, 53:190-197

5. Carter-Kent C, Zein NN, Feldstein AE: Cytokines in the pathogenesis of fatty liver and disease progression to steatohepatitis: implications for treatment. Am J Gastroenterol 2008, 103:1036-1042

6. Nestle FO, Di Meglio P, Qin JZ, Nickoloff BJ: Skin immune sentinels in health and disease. Nat Rev Immunol 2009, 9:679-691

7. Guilloteau K, Paris I, Pedretti N, Boniface K, Juchaux F, Huguier V, Guillet G, Bernard FX, Lecron JC, Morel F: Skin inflammation induced by the synergistic action of IL-17A, IL-22, oncostatin M, IL-1 \{alpha\}, and TNF-\{alpha\} recapitulates some features of psoriasis. J Immunol 2010, 184:5263-5270

8. Rabeony H, Petit-Paris I, Garnier J, Barrault C, Pedretti N, Guilloteau K, Jegou JF, Guillet G, Huguier V, Lecron JC, Bernard FX, Morel F: Inhibition of keratinocyte differentiation by the synergistic effect of IL-17A, IL-22, IL-1 $\alpha$, TNF $\alpha$ and oncostatin M. PLoS One 2014, 9:e101937

9. Dowlatshahi EA, Van Der Voort EA, Arends LR, Nijsten T: Markers of systemic inflammation in psoriasis: a systematic review and metaanalysis. Br J Dermatol 2013, 169:266-282

10. Targher G, Bertolini L, Rodella S, Lippi G, Franchini M, Zoppini G, Muggeo M, Day CP: NASH predicts plasma inflammatory biomarkers independently of visceral fat in men. Obesity 2008, 16:1394-1399

11. Targher G, Day CP, Bonora E: Risk of cardiovascular disease in patients with nonalcoholic fatty liver disease. N Engl J Med 2010, 363 : $1341-1350$

12. Gelfand JM, Neimann AL, Shin DB, Wang X, Margolis DJ, Troxel AB: Risk of myocardial infarction in patients with psoriasis. JAMA 2006, 296:1735-1741

13. Matsuzawa N, Takamura T, Kurita S, Misu H, Ota T, Ando H, Yokoyama M, Honda M, Zen Y, Nakanuma Y, Miyamoto KI, Kaneko S: Lipid-induced oxidative stress causes steatohepatitis in mice fed an atherogenic diet. Hepatology 2007, 46:1392-1403

14. Van der Fits L, Mourits S, Voerman JS, Kant M, Boon L, Laman JD, Cornelissen F, Mus AM, Florencia E, Prens EP, Lubberts E: Imiquimod-induced psoriasis-like skin inflammation in mice is mediated via the IL-23/IL-17 axis. J Immunol 2009, 182:5836-5845

15. Brunt EM, Kleiner DE, Wilson LA, Belt P, Neuschwander-Tetri BA: Nonalcoholic fatty liver disease (NAFLD) activity score and the histopathologic diagnosis in NAFLD: distinct clinicopathologic meanings. Hepatology 2011, 53:810-820
16. Liu Y, Lagowski JP, Gao S, Raymond JH, White CR, Kulesz-Martin MF: Regulation of the psoriatic chemokine CCL20 by E3 ligases Trim32 and Piasy in keratinocytes. J Invest Dermatol 2010, 130:1384-1390

17. Gisondi P, Targher G, Zoppini G, Girolomoni G: Non-alcoholic fatty liver disease in patients with chronic plaque psoriasis. J Hepatol 2009, 51:758-764

18. Roberts KK, Cochet AE, Lamb PB, Brown PJ, Battafarano DF, Brunt EM, Harrison SA: The prevalence of NAFLD and NASH among patients with psoriasis in a tertiary care dermatology and rheumatology clinic. Aliment Pharmacol Ther 2015, 41:293-300

19. Flutter B, Nestle FO: TLRs to cytokines: mechanistic insights from the imiquimod mouse model of psoriasis. Eur J Immunol 2013, 43:3138-3146

20. Van Belle AB, de Heusch M, Lemaire MM, Hendrickx E, Warnier G, Dunussi-Joannopoulos K, Fouser LA, Renauld JC, Dumoutier L: IL-22 is required for imiquimod-induced psoriasiform skin inflammation in mice. J Immunol 2012, 188:462-469

21. Abedini R, Salehi M, Lajevardi V, Beygi S: Patients with psoriasis are at a higher risk of developing nonalcoholic fatty liver disease. Clin Exp Dermatol 2015, 40:722-727

22. Debbaneh M, Millsop JW, Bhatia BK, Koo J, Liao W: Diet and psoriasis, part I: impact of weight loss interventions. J Am Acad Dermatol 2014, 71:133-140

23. Hebbard L, George J: Animal models of nonalcoholic fatty liver disease. Nat Rev Gastroenterol Hepatol 2011, 8:35-44

24. Wilson NJ, Boniface K, Chan JR, McKenzie BS, Blumenschein WM, Mattson JD, Basham B, Smith K, Chen T, Morel F, Lecron JC, Kastelein RA, Cua DJ, McClanahan TK, Bowman EP, de Waal Malefyt R: Development, cytokine profile and function of human interleukin 17-producing helper T cells. Nat Immunol 2007, 8:950-957

25. Ha HL, Wang H, Pisitkun P, Kim JC, Tassi I, Tang W, Morasso MI, Udey MC, Siebenlist U: IL-17 drives psoriatic inflammation via distinct, target cell-specific mechanisms. Proc Natl Acad Sci U S A 2014, 111:E3422-E3431

26. Tortola L, Rosenwald E, Abel B, Blumberg H, Schäfer M, Coyle AJ, Renauld JC, Werner S, Kisielow J, Kopf M: Psoriasiform dermatitis is driven by IL-36-mediated DC-keratinocyte crosstalk. J Clin Invest 2012, 122:3965-3976

27. Jhun JY, Yoon BY, Park MK, Oh HJ, Byun JK, Lee SY, Min JK, Park SH, Kim HY, Cho ML: Obesity aggravates the joint inflammation in a collagen-induced arthritis model through deviation to Th17 differentiation. Exp Mol Med 2012, 44:424-431

28. Kim HY, Lee HJ, Chang YJ, Pichavant M, Shore SA, Fitzgerald KA, Iwakura Y, Israel E, Bolger K, Faul J, DeKruyff RH, Umetsu DT: Interleukin-17-producing innate lymphoid cells and the NLRP3 inflammasome facilitate obesity-associated airway hyperreactivity. Nat Med 2014, 20:54-61

29. Zhang Y, Li Q, Rao E, Sun Y, Grossmann ME, Morris RJ, Cleary MP, Li B: Epidermal fatty acid binding protein promotes skin inflammation induced by high-fat diet. Immunity 2015, 42:953-964

30. Kim BS: Innate lymphoid cells in the skin. J Invest Dermatol 2015, $135: 673-678$

31. Sanmiguel JC, Olaru F, Li J, Mohr E, Jensen LE: Interleukin-1 regulates keratinocyte expression of $\mathrm{T}$ cell targeting chemokines through interleukin-1 receptor associated kinase-1 (IRAK1) dependent and independent pathways. Cell Signal 2009, 21:685-694

32. Beer HD, Contassot E, French LE: The inflammasomes in autoinflammatory diseases with skin involvement. J Invest Dermatol 2014, 134:1805-1810 